\title{
Sensitivity and uncertainty in flood inundation modelling - concept of an analysis framework
}

\author{
T. Weichel ${ }^{1}$, F. Pappenberger ${ }^{2}$, and K. Schulz ${ }^{1}$ \\ ${ }^{1}$ Helmholtz Centre for Environmental Research - UFZ, Dept. for Computational Landscape Ecology, Permoserstr. 15, 04318 \\ Leipzig, Germany \\ ${ }^{2}$ European Centre for Medium-Range Weather Forecasts, Shinfield Park, Reading, RG2 9AX, UK
}

Received: 15 January 2007 - Revised: 11 April 2007 - Accepted: 4 May 2007 - Published: 16 May 2007

\begin{abstract}
After the extreme flood event of the Elbe in 2002 the definition of flood risk areas by law and their simulation became more important in Germany. This paper describes a concept of an analysis framework to improve the localisation and duration of validity of flood inundation maps. The twodimensional finite difference model TrimR2D is used and linked to a Monte-Carlo routine for parameter sampling as well as to selected performance measures. The purpose is the investigation of the impact of different spatial resolutions and the influence of changing land uses in the simulation of flood inundation areas. The technical assembling of the framework is realised and beside the model calibration, first tests with different parameter ranges were done. Preliminary results show good correlations with observed data, but the investigation of shifting land uses reflects only poor changes in the flood extension.
\end{abstract}

\section{Introduction}

After the extreme flood event of the Elbe river in 2002 (DKKV, 2004) the German Water Resources Act was enhanced through the paragraphs $\$ 31 \mathrm{~b}$ and $\$ 31 \mathrm{c}$ (Bundesrepublik Deutschland, 2005). Because of a total loss of more than 9 billion euros in Germany a comprehensive localisation of the potential flood risk areas was finalised. In this approach the authors focus on two main points in the simulation of potential flooded areas. Besides the integration of detailed topographic data the influence of on-site land use changes are investigated. However, there are still major research challenges in the flood inundation modelling that need to be addressed like the problem of the spatial resolution and derivation of effective model parameterisations (Bates et al., 2006; Hengl 2005). Due to the general development

Correspondence to: T. Weichel

(thilo.weichel@ufz.de) of the airborne laser altimeter technology, detailed data with a spatial resolution lower than $1 \mathrm{~m}$ and a high horizontal and vertical accuracy $(<0.2 \mathrm{~m})$ are available for the hydrodynamic modelling. The increasing computer capabilities offer a better application of such large data volumes too. Therefore the use of these data in the flood inundation modelling with a detailed representation of the topography leads to the question of the required accuracy concerning the aspired results (Hengl, 2005). Furthermore the impact of distributed land use types described through roughness values is also discussed (Pappenberger et al., 2005; Werner et al., 2005). Whereas Werner et al. (2005) came to the result that the impact of distributed floodplain roughness values is restricted to the dominant land use. But how far land use changes, especially in urban areas, follow this result or show other tendencies will researched in this study. First historical and later possible future changes will be integrated in the simulation of inundation maps. If it's possible to achieve a sustained success with this approach the reliability of the simulated inundation areas could be improved. These limitations still introduce a significant amount of uncertainties into any prediction of potential flood risk thereby hampering any consequent planning or decision making process. Especially urban areas require effective and differentiated approaches for the modelling of flood risk areas due to the accumulation of monetary values (DKKV, 2004). The uncertainty and sensitivity analysis as methodical tools provide a general basis of model evaluation (Aronica et al., 2002; Crosetto et al., 2000; Pappenberger et al., 2005).

\section{Investigation site an available data}

Focussing on urban areas and because of the availability of detailed data there, a $25 \mathrm{~km}$ reach of the Elbe River in the city of Dresden is selected as investigation site (Fig. 1). The gauge Dresden in the centre of the city is used as reference.

Published by Copernicus Publications on behalf of the European Geosciences Union. 


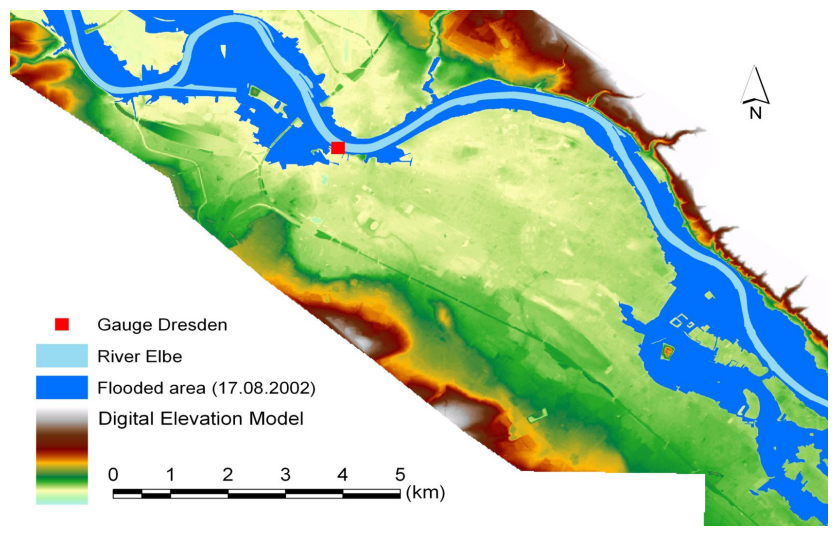

Fig. 1. Investigation site Dresden with flood extent (Environmental Agency Dresden).

Values of discharge and water level are hourly available by the Water and Shipping Authority. Tributaries are ignored. The used topographic data based on a digital elevation model (DEM), made by airborne laser scanning. The river channel is separate measured by the German Federal Institute of Hydrology. Both data are useable with a spatial resolution of one meter. The observed flood extension during the flood peak (17.08.2002) is prepared by airborne photographs and local watching. The distributed roughness is deduced from the "Biotoptypenkartierung" (scale 1:10.000). Further data like sandbag defence lines, building polygons or potential inundation maps by law are available by the local authority Environmental Agency as soon as the background information about the data.

\section{Objectives}

Based on the questions, how detailed flood inundation maps have to be and for what time period they are valid, the following objectives are addressed.

I. Evaluation of the spatial scale depended parameter sensitivity concerning to the quality of model predictions.

The purpose is the estimation of the most effective spatial resolution of the used parameters as well as the definition of their ranges relative to the required spatial scale (Bates et al., 2006; Hengl, 2006). Because different detailed inundation maps, like in spatial scale of 1:5000 or 1:100 000 should obtain an adapted spatial resolution of their simulation input, especially the DEM.

II. Analysis of the impact of land use changes, including their spatiotemporal dynamics of land surface properties on the modelling of flood inundation areas.

Here, the impact of land use changes concerning to the potential flood extension, especially in high structured and dynamic urban areas, is investigated. Based on the fact, that urbanisation is characterized by a development into the direction of floodplains (Fig. 2) the real inundated areas change accordingly. The roughness as a high sensitive parameter of the hydrodynamic modelling is used to detect possible correlations between land use changes and the changes of flooded areas.

\section{Method}

To obtain the mentioned objectives, a methodical approach of an analysis framework, which consists of a Monte-Carlo (MC) based sensitivity and uncertainty analysis, is prepared. This is a type of a sensitivity analysis (Saltelli et al., 2000), which performs multiple simulations with randomly selected model inputs for defined parameters. The current investigations are limited to the spatial resolution of the topography (DEM), the distributed roughness and the hydrograph. The hydrograph is also considered, because measured peak values during extreme flood events with a large return period are significant uncertain (Apel et al., 2004). The computational design is a combination of a MC routine to simulate the parameter inputs, the hydrodynamic model TrimR2D to compute the inundation areas and different performance measures to evaluate the quality of the modelled results (Fig. 3). Furthermore the approach is designed in a way that any parameter of the hydrodynamic model could be steered and investigated. Given the duration and required memory of the 2d-modelling the framework is implemented in a highperformance computer cluster with 64 CPUs. Depending on the results of the performance measures, the uncertainty and sensitivity analysis will be executed by a stepwise adaptation of the single parameter ranges.

\subsection{Monte-Carlo sampling}

The MC method to sample the model input is the first part of the framework, which apply a quasi random sampling routine after Sobol (1993). This procedure estimates a variancebased sensitivity index and computes a possible uniform distribution. The approach avoids randomly distributed patterns in the sampling of the parameter values. The reason for the selection of this routine is the consideration of the whole range of the chosen parameter space without any internal distribution. The results of the MC sampling are transferred into the selected parameter ranges. For example, the ranges of the parameter roughness are distributed accordingly to the number of different land use types and vary around their typical values. Depending on the number of samples the model will run.

\subsection{Hydrodynamic model TRIMR2D}

The raster-based hydrodynamic model TRIMR2D (Transient Inundation Model for Rivers-2 Dimensional) is applied in this study. It solves the two-dimensional depth-averaged shallow water equations for unsteady flow, which based on the conservation of mass (Eq. 1) and momentum (Eqs. 2 

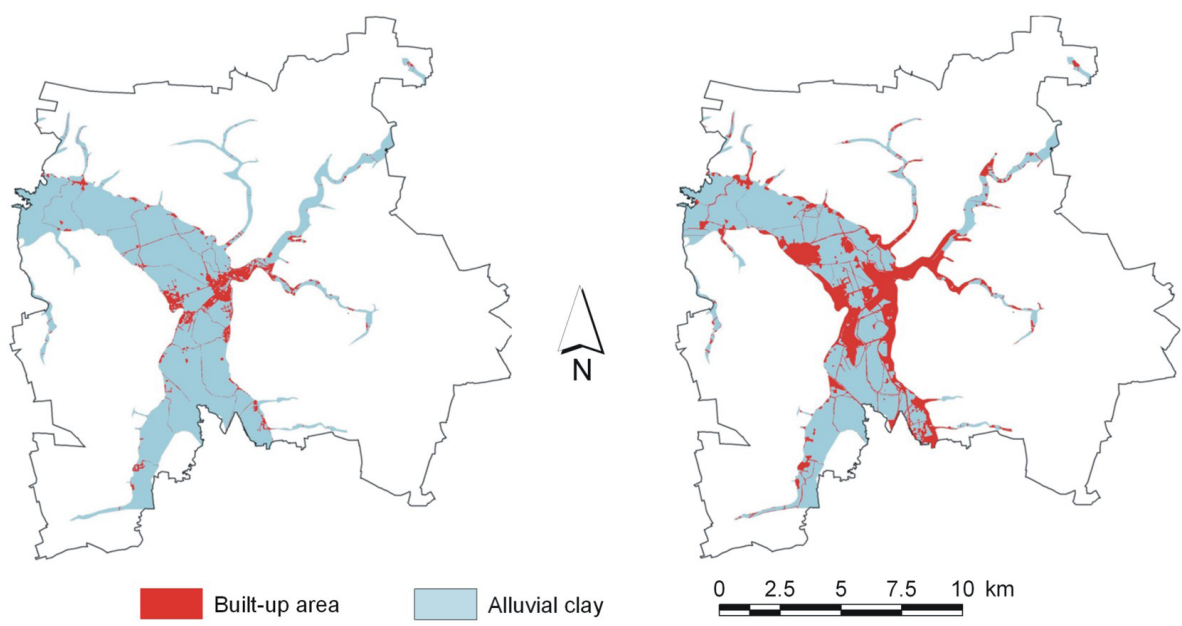

Fig. 2. State of the built-up area in the floodplain in the years 1870 (left) and 2003 (right) at the example of Leipzig, Germany.

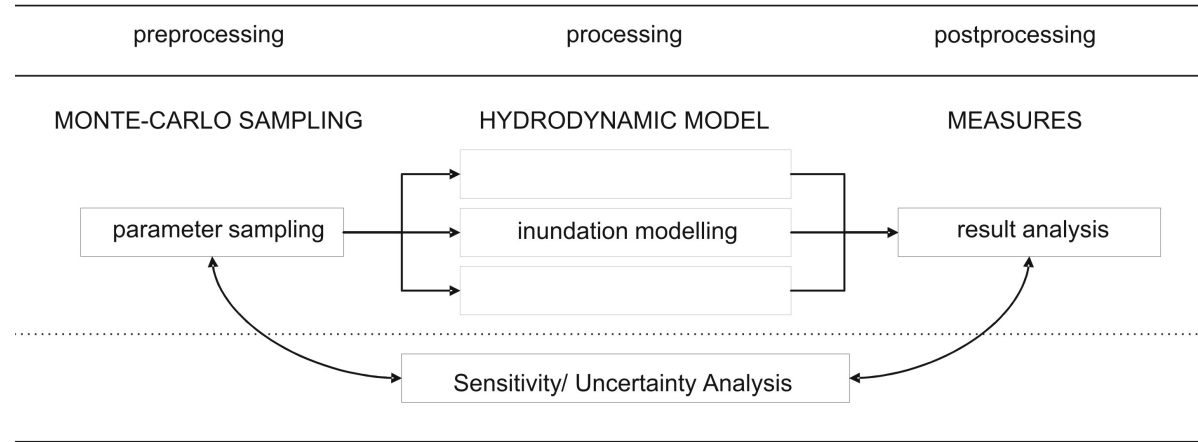

Fig. 3. Concept of the sensitivity and uncertainty framework.

and 3). The numerical solution uses a semi-implicit, semiLagrangian

$$
\begin{aligned}
& \frac{\partial \zeta}{\partial t}+\frac{\partial[(h+\zeta) u]}{\partial x}+\frac{\partial[(h+\zeta) v]}{\partial y}=0 \\
& \frac{\partial u}{\partial t}+u \frac{\partial u}{\partial x}+v \frac{\partial u}{\partial y}=-g \frac{\partial \zeta}{\partial x}+\frac{1}{\rho(h+\zeta)}\left(\tau_{x}^{w}-\tau_{x}^{b}\right)+A_{h} \nabla^{2} u+f v
\end{aligned}
$$

$$
\frac{\partial v}{\partial t}+u \frac{\partial v}{\partial x}+v \frac{\partial v}{\partial y}=-g \frac{\partial \zeta}{\partial y}+\frac{1}{\rho(h+\zeta)}\left(\tau_{y}^{w}-\tau_{y}^{b}\right)+A_{h} \nabla^{2} u+f u
$$

finite difference approach and applies a Manning-Chezy type expression as bottom-stress term (Eq. 4) with $\gamma$ as bed friction factor (Eq. 5).

$$
\begin{aligned}
& \frac{1}{\rho(h+\zeta)} \tau_{x}^{b}=\gamma u \text { and } \frac{1}{\rho(h+\zeta)} \tau_{x}^{b}=\gamma v \\
& \gamma=\frac{g \sqrt{u^{2}+v^{2}}}{C^{2}(h+\zeta)}
\end{aligned}
$$

A complete description of the used equations is detailed specified in the literature by Casulli (1990) and Fulford (2003). The TrimR2D code itself is a further development of a model, which was originally developed for coastal and estuarine environments (Casulli, 1990; Cheng et al., 1993). The performance of the model TrimR2D is documented for the uniform depth flows, laboratory dam-break flows and large-scale riverine flows (Fulford, 2003). The U.S. Geological Survey applies the model for near-real-time flood forecasting (USGS). The advantage of this model is the free access to the source code, which allows a flexible integration in the uncertainty framework. Because of the fact, that the roughness parameter was not spatial differentiated in the original source code, the authors upgraded the model concerning this functionality. Corresponding to the spatial resolution of the topographic input as a grid, the distributed roughness was also integrated in the code. The already existent part of the roughness term in the code was expanded by adding an array dimension for the distributed values into the appropriate variable. Figure 4 shows results of the calibration at the gauge Dresden during the time of the flood 
Table 1. Values of Mannings roughness $\left(\mathrm{m}^{1 / 3} \mathrm{~s}-1\right)$ in the model calibration.

\begin{tabular}{lll} 
& Type 1 & Type 2 \\
\hline Channel & 0.022 & 0.025 \\
& & 0.015 (pavement) \\
Floodplain & 0.044 & 0.033 (grassland, farmland) \\
& & 0.040 (fouling) \\
& & 0.050 (fouling) \\
& & 0.066 (woods, building) \\
\hline
\end{tabular}

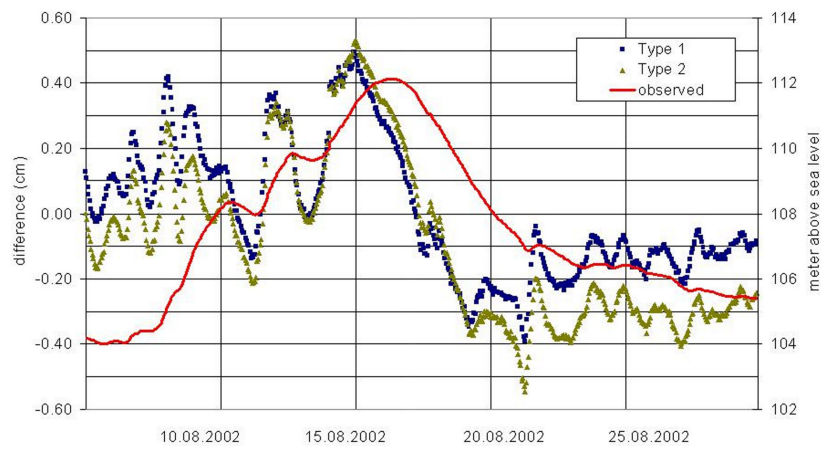

Fig. 4. Model calibration at the gauge Dresden with two different sets of roughness values (Table 1). Differences are calculated between the observed data and simulated results.

2002. Concerning to the roughness, two different types are applied (Table 1), where type 1 is the best parameter set after Werner et al. (2005) and type 2 an aggregated spatial distributed set based on the Biotoptypenkartierung. The spatial resolution was set by a cellsize of $20 \mathrm{~m}$. Therefore the differences between the observed and simulated data in Fig. 4 are acceptable in consideration of the uncertainties of the DEM, the spatial resolution as well as the measured values at peak are considered.

\subsection{Performance measures}

The purpose of performance measures in the evaluation of inundation modelling results is the comparison of simulated and observed flood extents. This methodical approach is commonly used in the evaluation of spatial predictions in meteorological models (Pappenberger, 2007) and also applied in the current inundation modelling (Aronica et al., 2002; Hunter, 2005; Pappenberger et al., 2007). The comparison based on a reducing of the observed and simulated data to spatially distributed discrete binary patterns with similar extent and cell size. Then the correctly and incorrectly classified dry and wet areas are identified by a contingency table (Table 2) and transferred in the performance measures. More complex classifications than the binary will not applied in this study. The used performance measures, which are detailed described in the literature (Aronica et al., 2002; Hunter, 2005; Pappenberger et al., 2007), differ in their kind
Table 2. Classification of the input of the performance measures resulted by an contingency table for the binary result pattern.

\begin{tabular}{llcr}
\hline \multirow{2}{*}{ Contingency table } & \multicolumn{2}{c}{ Observed } \\
& & Inundated & Not inundated \\
\hline \multirow{3}{*}{ Simulated } & Inundated & $\mathrm{a}$ (hits) & $\mathrm{b}$ (fales) \\
& Not inundated & $\mathrm{c}$ (misses) & $\mathrm{d}$ (zero) \\
\hline
\end{tabular}

of identification. Equation (6) returns a simple overall accuracy index of the correctly modelled values. But in case of an investigated domain, which is large in relation to the possible flood extent, the measure tends to an overestimation. To amplify the analysis of parameter sensitivity and model uncertainty the well described measures in Eqs. (7) and (8) (Aronica et al., 2002; Hunter, 2005) are applied too. Equation (7), also known as the threat score or critical success index, applies only the correctly predicted fraction of observed and/or modelled events. It penalises both the false and misses (Table 2) and therefore it's prone to an over-prediction of the modelled results (Hunter, 2005). Therefore Eq. (8) is also used, which penalise additionally over prediction of the flood extent through the integration of the failed predictions in the numerator (Hunter 2005).

$$
F^{<1>}=\frac{\sum_{i=1}^{n} a+\sum_{i=1}^{n} d}{n} \quad \text { (Aronica et al., 2002) }
$$

$$
\begin{aligned}
& F^{<2>}=\frac{\sum_{i=1}^{n} a}{\left(\sum_{i=1}^{n} a+\sum_{i=1}^{n} b+\sum_{i=1}^{n} c\right)} \quad \text { (Aronica et al., 2002) } \\
& F^{<2>}=\frac{\sum_{i=1}^{n} a-\sum_{i=1}^{n} b}{\left(\sum_{i=1}^{n} a+\sum_{i=1}^{n} b+\sum_{i=1}^{n} c\right)} \quad \text { (Hunter, 2005) }
\end{aligned}
$$

The maximum flood extent during a water level of $9.24 \mathrm{~m}$ at the gauge Dresden is used as observed pattern. Uncertainties in the defined shoreline are not yet included in the preliminary investigations, because of the detailed recording of the data.

\section{Preliminary results}

The technical realisation of the analysis framework is done. Besides the calibration, first results are error plots, like in Fig. 5, which represent a form of Eq. (6). Here, 2000 simulations with varying values for channel and floodplain roughness are compared at the flood peak with the observed data in a binary approach of wet and dry cells. But it's only useful 
Table 3. Nomenclature in the used equations.
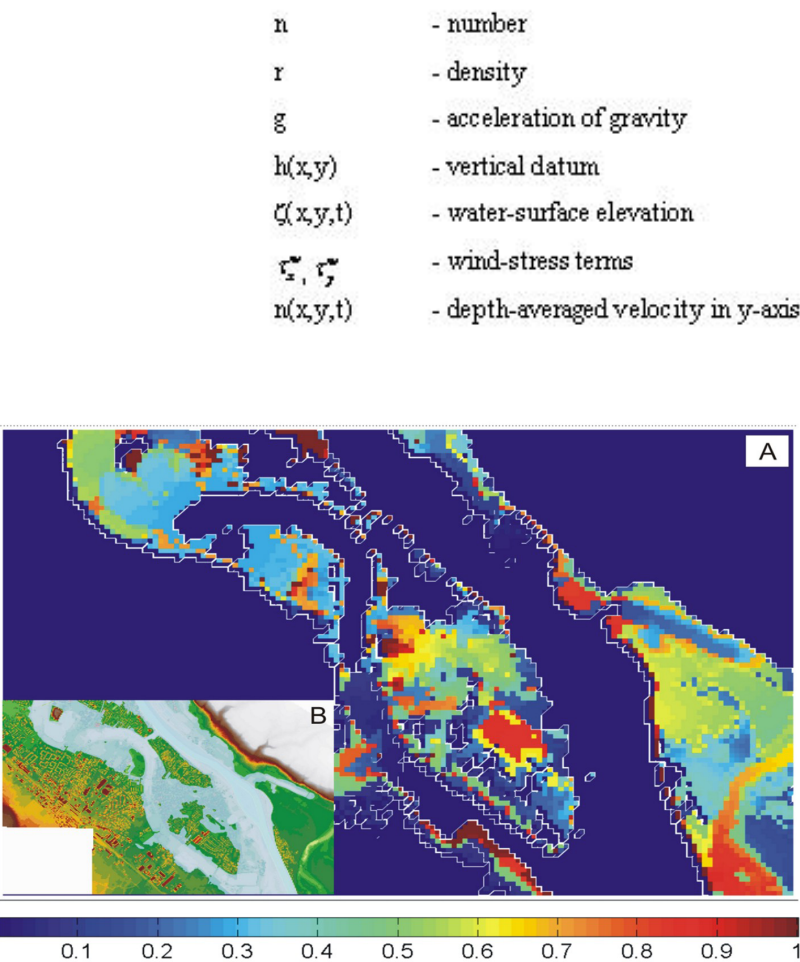

Fig. 5. The error plot (A) represents a spatial classification of the simulation quality with a diminishing performance from 0 to 1 . The reference map (B) is an overview of the domain (DEM) with the observed inundation area (light blue).

to an identification of certainly or uncertainly simulated areas without information about the responsible parameter set. For this purpose the mentioned performance measures will be analysed in diagrams like Fig. 6. Based on these result the real investigation of the aspired objectives will be realised in the next steps of the study. Especially the edges of the maximum flood extend in areas with buildings and urban infrastructures are in the focus of the further research.

\section{Conclusions}

The development and testing of an analysis framework to investigate the impact of sensitivity and uncertainty in the flood inundation modelling is described. It's a combination of a MC routine to simulate the parameter inputs, the hydrodynamic model TrimR2D and different performance measures to evaluate the modelled results. The objectives are the analysis of the impact of different spatial distributed model inputs concerning to the reliability of the modelled results, especially in urban areas. The single parts of the framework are explained as well as their functionality in this approach, which result in a sensitivity and uncertainty tool.
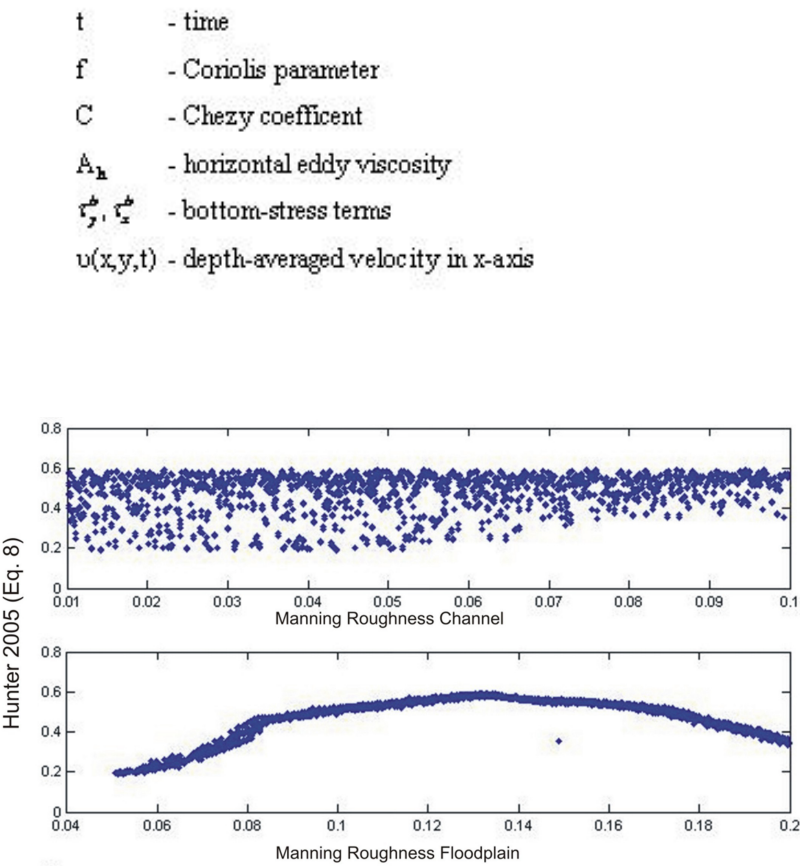

Fig. 6. Performance of Eq. (6) in a first general analysis of the distributed roughness.

Acknowledgements. The author thanks D. Haase for the preparation of the land use data.

Edited by: K.-E. Lindenschmidt

Reviewed by: H. Bormann and M. Fink

\section{References}

Apel, H., Thieken, A. H., Merz, B., and Blöschl, G.: Flood risk assessment and associated uncertainty, Nat. Hazards Earth Syst. Sci., 4, 295-308, 2004, http://www.nat-hazards-earth-syst-sci.net/4/295/2004/.

Aronica, G., Bates, P. D., and Horritt, M. S.: Assessing the uncertainty in distributed model predictions using observed binary information within GLUE, Hydrol. Processes, 16, 2001-2016, 2002.

Bates, P. D., Wilson, M. D., Horrit, M. S., Mason, D. C., Holden, N., and Currie, A.: Reach scale floodplain inundation dynamics observed using airborne synthetic aperture radar imagery: Data analysis and modelling, J. Hydrol., 328, 306-318, 2006.

Bundesrepublik Deutschland: Wasserhaushaltsgesetz (WHG), Letzte Änderung durch: 25. Juni 2005 (BGB1. I S. 1746, 1756), 2005.

Casulli, V.: Semi-implicit finite difference methods for the twodimensional shallow water equations, Journal of Computational Physics, 86(1), 56-74, 1990.

Cheng, R. T., Casulli, V., and Gartner, J. W.: Tidal, Residual, Intertidal Mudflat (TRIM) Model and its applications to San Fran- 
cisco Bay, Estuarine, Coastal and Shelf Science, 36, 235-280, 1993.

Crosetto, M., Tarantola, S., and Saltelli, A.: Sensitivity and uncertainty analysis in spatial modelling based on GIS, Agriculture, Ecosystems \& Environment, 81, 71-79, 2000.

DKKV: Flood risk reduction in Germany, DKKV publication, 29e, 2004.

Fulford, J. M.: Computational Technique and Performance of Transient Inundation Model for Rivers - 2 Dimensional, USGS OpenFile Report 03-371, 2003.

Hengl, T.: Finding the right pixel size, Computer \& Geosciences, 32, 1283-1298, 2006.

Hunter, N. M.: Utility of different data types for calibrating flood inundation models within a GLUE framework, Hydrol. Earth Syst. Sci., 9, 412-430, 2005,

http://www.hydrol-earth-syst-sci.net/9/412/2005/.

Pappenberger, F., Beven, K., Horritt, M., and Blazkova, S.: Uncertainty in the calibration of effective roughness parameters in HEC-RAS using inundation and downstream level observations, J. Hydrol., 302, 46-69, 2005.
Pappenberger, F., Frodsham, K., Beven, K., Romanowicz, R., and Matgen, P.: Fuzzy set approach to calibrating distributed flood inundation models using remote sensing observations, Hydrol. Earth Syst. Sci., 11, 739-752, 2007, http://www.hydrol-earth-syst-sci.net/11/739/2007/.

Saltelli, A., Chan, K., and Scott, E. M.: Sensitivity analysis, Wiley, 2002.

Sobol, I. M.: Sensitivity analysis for nonlinear mathematical models, Math. Model. Comput. Exp, 1, 407-414, 1993.

USGS: http://pubs.usgs.gov/fs/2004/3060/pdf/fs20043060.pdf, 11.04.2007.

Werner, M. G. F., Hunter, N. M., and Bates, P. D.: Identifiability of distributed floodplain roughness values in flood extent estimation, J. Hydrol., 314, 139-157, 2005. 